\title{
SYSTEME D'EDUCATION ISLAMIQUE EN FRANCE ET EN INDONESIE
}

\author{
Valérie Grollau \\ 2 Alle De Moncontour 86000 POITIERS FRANCE \\ Email : valerawan@yahoo.fr
}

\section{Qiqi Yuliati Zaqiah}

Tunas Unggul, Jl. Pasir Impun No. 94 Kec. Mandalajati Bandung

Email : qiqi_uwe@yahoo.com

\section{RÉSUMÉ}

Cette étude a pour but d'examiner la position de l'enseignement islamique qui est appliqué en Indonésie actuellement, en comparant les systèmes éducatifs en France et en Indonésie. A partir de cette étude, nous espérons montrer que les innovations du système d'enseignement islamique qui sont réalisées de manière continue, le sont dans le but d'améliorer la qualité du système éducatif en Indonésie. Le système éducatif en France est très avancé et possède des institutions de premier plan et innovatives dans différents domaines. Cela s'explique par l'engagement du gouvernement français qui depuis l'année 2004 attribue environ $23 \%$ de son budget total à l'éducation. Le niveau de vie des professeurs en France est classé au 3ème rang des fonctionnaires. Bien que le nombre des institutions d'enseignement islamique en France soit encore très réduit, leur gestion a un standard très élevé, comme l'ensemble de l'enseignement en général en France. Tandis que les institutions islamiques en Indonésie sont très nombreuses, mais leur gestion est encore très variée et la majorité ne sont pas conformes au standard national. Cependant, l'enseignement islamique en Indonésie a grandit de manière exponentielle dans l'histoire de l'enseignement national et a apporté une contribution significative dans le développement du système de l'éducation nationale

Mots clés: Comparaison d'enseignement, France, Indonésie

\begin{abstract}
ABSTRAK
[Penelitian ini bertujuan untuk menguji posisi ajaran Islam yang saat ini diterapkan di Indonesia, membandingkan sistem pendidikan di Perancis dan Indonesia. Dari penelitian ini, kami berharap dapat menunjukekan bahwa inovasi dari sistem pendidikan Islam yang dilakukan secara terus menerus, dalam rangka meningkatkan kualitas pendidikan di Indonesia. Sistem pendidikan di Perancis sangat maju dan memiliki lembaga-lembaga terkemuka dan inovatif di daerah yang berbeda. Hal ini mencerminkan komitmen pemerintah Perancis sejak tahun 2004 mengalokasikan sekitar 23\% dari total anggaran untuk pendidikan. Standar bidup guru di Perancis berada di peringkat ke-3 di antara pejabat publik. Meskipun jumlah lembaga pendidikan Islam di Prancis masib minimal, manajemen memiliki standar yang sangat tinggi, karena semua pendidikan secara umum di Perancis. Sementara lembaga-lembaga Islam di Indonesia sangat banyak, tapi manajemen mereka masib sangat bervariasi dan sebagian besar tidak sesuai dengan standar nasional. Namun, pendidikan Islam di Indonesia telah berkembang
\end{abstract}


pesat dalam sejarah pendidikan nasional dan telah membuat kontribusi yang signifikan dalam pengembangan sistem pendidikan nasional].

Kata Kunci: Pendidikan, Perancis, Indonesia

\section{INTRODUCTION}

Une étude comparative est une étude dont l'essence est de comparer. Le but de cette étude est d'étudier les ressemblances et les différences entre deux ou plusieurs faits et les caractéristiques de l'objet qui est étudié dans un cadre bien déteminé. Selon Nazir, une étude comparative est une sorte d'étude descriptive qui veut répondre de manière fondamentale à la relation de cause à effet, en analysant les facteurs responsables de la réalisation ou de l'apparition d'un phénomène défini. Donc, une étude comparative est une sorte d'étude utilisée pour comparer deux catégories ou plus selon une variable précise (Nazir, 2005, p. 58).

Une étude comparative dans le domaine de l'éducation peut être réalisée en comparant les systèmes éducatifs appliqués dans deux endroits différents. Le but d'une étude comparative dans le domaine de l'éducation est de comparer divers aspects d'un système d'éducation y compris les antécédants qui ont influencé le dit-système et cela s'explique par le fait qu'une décision sera très influencée par différents antécédants, comme par exemple des mesures politiques dépendantes du gouvernement au pouvoir.

Le but d'une étude comparative dans le domaine de l'éducation est de permettre d'examiner la position actuelle de l'enseignement dans notre pays, car l'enseignement va progresser, si les experts ou les acteurs de l'enseignement veulent apprendre et observer comment se déroule l'enseignement dans d'autres pays. Ceci reprend ce que dit Harold J. Noah, qu'en comparant l'enseignement de diverses nations, nous pouvons obtenir des renseignements sur l'état de l'enseignement dans d'autres pays ou régions. En connaissant la situation de l'enseignement dans beaucoup de pays ou régions, nous pouvons profiter de ce savoir pour l'appliquer dans l'élaboration de l'enseignement dans notre propre pays (Rohman, 2010, p. 100).

C'est pourquoi nous essayons, à travers cette opportunité d'effectuer une comparaison entre le système d'éducation islamique en Indonésie avec un autre pays, plus particulièrement la France. Nous effectuons cette étude sur la base des considérations que, premièrement la communauté musulmane française est assez importante comparée aux autres pays européens, c'est-àdire environ 6 millions de musulmans. Deuxièmement, l'aspect historique de ce pays. La France est une unité politique qui a été unie par la domination de la Rome antique, où les Romains avaient un rôle très important pour le développement de la science, en particulier en matière d'éducation. De plus, 
en regardant les similitudes dans la politique et le gouvernement entre la France et l'Indonésie occupés, avaient un fort désir d'échapper aux entraves des envahisseurs. La rébellion a été déclenchée dans les deux pays par un texte écrit par des personnages très préocuppés par l'éducation, c'est-à-dire Rebelains (France) et Ki Hajar Dewantoro (Indonésie). Cela est soutenu également par la prise de conscience de l'importance de l'éducation dans la construction d'une nation de meilleur qualité (http://susantnext.blogspot.com /2012/02/. téléchargé le 1 Mai 2014).

\section{DISCUSSION}

\section{L'enseignement islamique en Indonésie \\ But de l'enseignement}

L'enseignement islamique en Indonésie est très dynamique et subit régulièrement des changements en fonction des orientations et des défis de notre siècle. Comme le dit Fazhur Rahman, l'enseignement islamique, à la base, c'est comment créer un enseignement capable d'augmenter la productivité intelectuelle de manière créative dans tous les domaines intelectuels en conservant un lien solide avec l'Islam (Rahman 1982, p. 134). C'est pourquoi, les valeurs islamiques doivent être l'esprit qui illumine tous les domaines intelectuels.

L'enseignement islamique possède des racines intelectuelles et une culture spécifique. Par exemple, (le madrasa) en Indonésie est la convergence réussie entre l'enseignement islamique classique et l'enseignement islamique moderne. Pourtant cette intégration subit toujours des changements dynamiques. Fazlur Rahman considère encore cette intégration comme un moyen défensif pour sauvegarder la manière de penser des musulmans de la contamination ou de la dépravation provenant des idées occidentales qui sont transmises à travers divers sciences, principalement les idées qui outrepassent les standards de la moralité des musulmans.

Dans cette panique spirituelle, la stratégie développée de manière universelle, à travers le monde musulman, est une stratégie qui semble mécanique: comment relier certaines nouvelles matières avec des matières anciennes pour obtenir un mélange qui soit plus stimulant (Fazlur Rahman 1982, p. 86).

Cependant, l'enseignement islamique en Indonésie possède une orientation d'équilibre en tant que manifestation de l'objectif de l'enseignement, qui s'explique parce que le but de la vie des hommes à travers le Coran est de développer les capacités essentielles des hommes de telle sorte que toutes les sciences qu'ils vont étudier par la suite vont fuser avec leur personnalité et leur créativité. L'orientation de ce genre d'enseignement se reflète dans le programme d'enseignement qui intègre les sciences 
religieuses avec les sciences générales de manière exhaustive. Car à la base, les sciences sont intégrées et ne peuvent pas être séparées (Rahman 1982, p. 323).

Selon Meuleman "L'enseignement islamique en Indonésie est destiné à conserver et enseigner la religion musulmane à travers ses aspects rituels, doctrinaires, sociaux, et culturels. Le Pesantren (l'internat) a été longtemps l'institution principale pour atteindre cet objectif. Bien que le pesantren a été influencé à différents niveaux par la modernisation et le changement, son influence sur la tradition éducative et sociale est encore assez forte, ». Alors qu'en Europe, l'Islam est étudié comme une science, par les non-musulmans à des fins scientifiques, commerciales, politiques et missionaristes. Cette approche de l'Islam s'est développée depuis le Moyen-Age, depuis qu'il existe une interaction entre les musulmans et les européens. Cependant l'augmentation récente de la communauté musulmane en Europe de l'Ouest et Centrale a fait naitre une nouvelle forme d'institutions islamiques. La forme de ces institutions diffère selon les pays avec divers facteurs.

\section{But de l'enseignement}

Dans la perspective de l'homme en tant que créature éduquée, nous dirigeons le but de l'éducation vers la réalisation du développement de la personnalité des hommes de manière équilibrée, aussi bien physique, social, intelectuel que spirituel. C'est ce que décrit al-Syaibany comme le but final de l'enseignement islamique qui essaye de développer les dispositions de l' élève, que ce soit l'esprit, le physique, la volonté et l'intelligence de manière dynamique, jusqu'à se construise une personnalité complète et qui soit propice à remplir son rôle en tant que califat sur terre (Langgulung, p. 67). Alors que Ibnu Miskawih a tendance à orienter le but de l'enseignement sur la morale qui permet de faire naitre de manière spontanée l'accomplissement de bonnes actions pour atteindre la perfection et obtenir le bonheur parfait. (as-sa'ádah) (Suwito, 2004, p. 116).

Cet objectif a un sens, qui est que l'enseignement islamique passe par le développement d'une vraie personnalité musulmane qui se soumet à Dieu et accomplit sa volonté, en conformité avec les règles de l'Islam, en remplissant son devoir sur terre et en faisant de la vie éternelle le but essentiel de son enseignement.

Cette conception décrit, de manière générale, qu'il y a deux choses qu’il faut réaliser dans la pratique de l'enseignement islamique, c'est 'la dimension dialectique horizontale' et 'la dimension de soumission verticale'. Dans la dimension dialectique horizontale, l'enseignement islamique doit pouvoir développer les réalités de la vie, aussi bien en ce qui concerne notre propre personne, la vie en société que l'univers. Alors que la dimension de soumission verticale 
décrit que l'enseignement islamique, en plus d'être un moyen pour entretenir, utiliser et conserver les ressources naturelles, doit aussi permettre d'enseigner les phénomènes et les mystères de la vie pour établir une relation éternelle avec le Créateur.

En pratique, Muhammad Athiyah al-Abrasyi conclut que le but de l'enseignement islamique se fonde sur cinq objectifs, qui sont : a) former une morale noble: b) préparer à la vie sur terre et à la vie dans l'au-delà; c) préparer à subvenir à ses besoins, à utiliser et à entretenir les avantages des richesses naturelles; d) faire naitre l'esprit scientifique chez les élèves; e) préparer des travailleurs professionnels et qualifiés (Zakiy al-Kaaf, 2003, p. 13-16).

Le deuxième Congrès International sur l'enseignement islamique qui a eut lieu en 1980 a Islamabad au Pakistan stipule que: "Le but de l'enseignement islamique est de faitre naitre un équilibre dans la personnalité des hommes de manière globale et équilibrée en entrainant l'esprit, l'intelligence et la réflexion (intelectuelle), des humains de manière rationnelle; les sentiments et les sens. C'est pourquoi, l'enseignement doit couvrir l'ensemble des aspects du caractère des élèves; l'aspect spirituel, intellectuel, imaginaire, physique, scientifique et langagier, aussi bien de manière individuelle que collective, et pousser à développer tous ces aspects dans la voie de la bonté et de la perfection. Le dernier objectif de l'enseignement islamique réside dans l'accomplissement de la soumission parfaite à Allah, de manière individuelle, communautaire et universelle (Langgulung, 1980, p. 206-207).

\section{Programme d'enseignement scolaire}

D’une manière générale, le système d'enseignement qui est utilisé en Indonésie est démocratique, bien que dans son application il y a souvent différents changements dans le programme d'enseignement et l'exécution de l'enseignement en lui-même. Depuis le début de l'indépendance jusqu'à aujourd'hui, l'Indonésie a changé plusieurs fois de programme d'enseignement. A savoir, pendant la période 1945-1949, a été publié le programme d'enseignement 1947. De 1950 à 1961, a été déterminé le programme d'enseignement 1952. Le programme d'enseignement de l'ordre ancien est le programme d'enseignement de 1964, alors que pour l'ordre nouveau on a établit le programme d'enseignement de 1994, KBK (le programme d'enseignement basé sur les compétences) puis l'amélioration du KBK est le KTSP, et enfin, le dernier né qui est à l'essai est le programme d'enseignement 2013.

Ces différents changements sont effectués dans le but d'améliorer l'enseignement actuel en Indonésie, et la base des changements est bien sur, 
fondée sur des études comparatives effectuées par les experts dans différents pays développés. Les changements qui sont jugés importants dans le programme d'enseignement de 2013 portent sur quatre normes de l'enseignement qui sont le standard d'admission, le standard du contenu, le standard du processus et le standard de la notation Ces quatre standards sont suffisament importants dans le changement du processus d'enseignement d'une manière générale.

Les changements du programme d'enseignement qui sont régulièrement effectués par le gouvernement, ont pour but d'améliorer la qualité de l'enseignement, pour que les diplomés puissent être compétitifs au niveau international. Les améliorations de la qualité du système d'éducation sont effectuées de manière complète et pas seulement dans l'enseignement formel mais également dans l'enseignement informel, et pas seulement dans les établissements scolaires dépendants du Ministère de l'éducation nationale mais également auprès des établissements dépendants du Ministère des religions, comme par exemple les madrasa (écoles coraniques).

L'étimologie di terme "curriculum" provient du latin, à savoir curro ou currere et ula ou ulums qui veut dire "racecourse" (champ/course de chevaux, distance de course, concours, course de voitures, circulation, rotation, stade, voiture de course (Ebster, 1980, p. 231). En accord avec l'évolution du monde de l'éducation, le concept du programme d'enseignement subit également des changements et des modifications du sens, du contenu au processus de l'enseignement comme le dit Robin: "The commonly accepted definition on the curriculum has changed from content of courses of study and list of subyects and course to all experiences which are offered to learners under the auspices or direction of the school" (Forgarty, 1991, p. 97).

Le concept du programme d'enseignement dans la loi sur le système de l'éducation nationale de 2003 article 1 alineas 11: dit que le programme d'enseignement est un ensemble de plans et de règles sur le contenu et les matières d'enseignement ainsi que les moyens utilisés comme guide pour organiser les activités d'enseignement (UU SPN, No. 20 Tahun 2003). Dans la perspective de l'enseignement islamique, le programme d'enseignement est la conceptualisation de la place et du rôle de l'homme en tant que serviteur de Allah et de califat sur terre, qui recouvre l'ensemble du potentiel humain (Irfan et Mastuki HS, p. 151).

Le programme d'enseignement est le fondement qui est utilisé par les éducateurs pour guider les élèves vers l'enseignement désiré à travers une accumulation de savoirs, de compétences et de comportements. De plus, les principes qui sont développés dans le curriculum d'enseignement islamique sont, entre autres ; (1) Orientation vers un objectif d'enseignement d'après le Coran, c'est-à-dire le développement des capacités essentielles de l'homme : 
intellectuelles, morales et spirituelles. (2) Intégrer les sciences religieuses et les sciences à caractère général de manière organisée et complète. (3) Pertinent, dans le sens qu'il peut apporter des ressources aux élèves leur permettant de s'adapter aux exigences de la vie moderne. (4) Fonctionnel, dans le sens qu'il peut pousser la productivité intelectuelle en conservant une relation étroite avec l'Islam (al-Nahlawi, 1878, p. 177-179).

Pour concrétiser un programme d'enseignement qui respecte les principes cités ci-dessus, il ne faut plus qu'il y ait de dichotomie entre la science religieuse et les sciences de connaissances générales. Ce point de vue est pratiquement similaire avec celui exprimé par Azra sur le fait que la réorientation du programme d'enseignement est faite en fonction de la façon de penser qui est séparée d'une croyance iréelle où l'amélioration du programme d'enseignement est uniquement basé sur les carences d'une partie de la population. D'un autre coté,le programme d'enseignement de l'enseignement islamique qui est orienté sur les valeurs, doit donner une direction et un but uniques pour que ce programme d'enseignement soit pertinent et fonctionnel et puisse répondre aux besoins nationaux, régionnaux et globaux (Azra, 1998, p. 26).

De sorte que le programme d'enseignement de l'enseignement islamique en Indonésie est un mélange entre un programme d'enseignement humaniste et un programme d'enseignement de reconstruction sociale. D'un côté, il faut engendrer des individus qui soient croyants, intelligents et créatifs. D'un autre coté, il faut que les jeunes diplômés aient des compétences et des ressources pour s'adapter aux exigences de la vie qui évoluent sans cesse, c'est ce que l'on nomme (des êtres parfaits). En observant l'aspect du programme d'enseignement dans l'enseignement islamique, il n'est donc pas possible de résoudre le problème uniquement par une expansion linéaire du système d'enseignement existant et l'ajustement pédagogique et technique de la réforme de l'éducation islamique est de créer un enseignement pour encourager la productivité intellectuelle dans tous les domaines de la création tout en conservant un attachement sérieux à l'Islam (Rahman, 1987. p. 134). Ainsi, les valeurs de l'Islam devraient être l'esprit qui caractérise l'ensemble du champ intellectuel.

Il y a plusieurs critères qui doivent être respectés dans le programme d'enseignement islamique selon (Al-Nahlawi, 1989, p. 273-277) ce sont: 1) Le système et l'élaboration des programmes devraient être en harmonie avec la nature humaine de manière à avoir la possibilité de la purifier, de la protéger des déviations et de la sauver; 2) Le curriculum doit s'attacher à atteindre l'objectif ultime de l'éducation islamique, qui est la sincérité, l'obéissance et l'adoration de Dieu, en plus de la réalisation des objectifs d'aspect psychologique, physique, social, culturel et intellectuel; 3) Le phasage 
et la spécification du programme d'enseignement devraient envisager la périodisation de l'évolution des apprenants ainsi que leur l'unicité (spécificité), en particulier les caractéristiques des enfants, et le sexe (masculin et féminin); 4) Les différentes applications, activités, exemples et textes existants dans le programme d'enseignement doivent maintenir à la fois les besoins de la vie réelle de la population tout en s'appuyant toujours sur les idéaux islamiques, comme la gratitude et l'estime de soi en tant que musulmans; 5) L'ensemble de la structure et de l'organisation du programme d'enseignement ne doivent pas être contradictoires et ne doivent pas s'opposer au mode de vie Islamique; 6) Le programme doit être réaliste ou doit pouvoir être appliqué conformément aux circonstances et conditions de vie d'un pays en particulier; 7) Les méthodes d'enseignement/apprentissage dans le programme doivent être flexibles et pouvoir être adaptées à diverses situations et conditions comme les différences individuelles, les intérêts et la capacité des élèves à comprendre et à exploiter la matière étudiée ; 8) Le programme doit être efficace dans le sens qu'il doit être rempli de valeurs éducatives pour façonner une attitude islamique dans la personnalité de l'enfant; 9) Le programme doit tenir compte des aspects "Amaliah" du comportement islamique, tel que l'éducation pour le jihad (la guerre sainte) et la da'wah islamique (la propagation de la foi) ainsi que l'implantation d'une communauté musulmane dans l'environnement scolaire.

Les caractéristiques du programme d'enseignement islamique comme il a été décrit ci-dessus ne place pas uniquement les élèves en tant qu'objets, mais aussi en tant que sujets qui doivent se cultiver de manière continue pour acquérir une maturité en concordance avec les concepts de l'Islam. On voit ici la spécificité du programme de l'enseignement islamique qui considère l'élève comme un être capable de progresser par lui-même à travers diverses activités éducatives. Les éducateurs et tous les autres composants de l'éducation, y compris les programmes d'études sont des médias ou des moyens qui doivent fournir une situation et des conditions favorables au prosessus de développement de la totalité des capacités possédées par les apprenants pour atteindre une perfection optimale.

\section{Les institutions de l'éducation}

Les écoles coraniques (madrasa) sont une manifestation de la volonté de la population en Indonésie pour répondre aux besoins de l'éducation, en particulier de la communauté musulmane. D’un aspect culturel, le madrasa est une sous-culture d'un système social où la société croit et se développe. Par conséquent, la majorité des madrasa en Indonésie, en particulier les madrasa privées-naissent généralement à l'initiative de la communauté locale. Toutefois, dans le développement ultérieur, les madrasa ont changé leur 
statut en devenant des établissements publiques et en suivant entièrement le système de l'éducation nationale. Les madrasa deviennent ainsi un instrument formel qui participe au changement sociétal en fonction de l'orientation de l'éducation nationale (Hasanah, 2010, p. 2).

Sur le plan historique, les madrasa sont essentiellement nées du processus de modernisation des établissements d'enseignement traditionnels dans un effort de répondre à la demande de la population qui est le support de l'existence des madrasa. Elles représentent l'évolution d'un des modèles éducatifs traditionnels musulmans indonésien, qui est le pesantren. Les madrasa se développent généralement dans l'entourage des pesantrens-et beaucoup d'entre eux sont l'évolution d'un model d'éducation-d'un modèle de pesantren pur en madrasa. Selon Karel Steenbrink, un madrasa est un renouvellement d'un système d'éducation traditionnelle islamique en apportant un nouveau contenu dans le prosessus d'apprentissage.

À bien des égards, les pesantrens qui s'étaient développées depuis la fin du 17ème siècle dans l'archipel, sont les précurseurs des établissements d'enseignement, les madrasa. Les recherches historiques montrent même que le modèle d'éducation des pesantren a un lien historique assez fort avec le système éducatif traditionnel en Inde qui s'est développé depuis des centaines d'années avant l'ère chrétienne. Quand les musulmans ont commencé à explorer l'archipel et à développer ce modèle, l'éducation «précurseur du madrasa" a commencé à être mis en œuvre dans les lieux de prière ou les petites mosquées situées dans les communautés locales (Dhofier, 1982).

C'est pourquoi, les madrasa adhèrent encore à la culture des pesantren, à la fois en terme de substance de l'enseignement ou dans la gestion de sa direction, la façon de diriger un madrasa est encore fortement influencée par les modèles des pesantren qui sont basés sur le modèle de leadership charismatique. Et même dans certains pesantren, le directeur est nommé directement par le kyai (titre religieux) en fonction de ses qualifications subjectives. Cela est principalement basé sur l'hypothèse que l'existence de madrasa complète l'éducation dispensée par les pesantren. La survie des madrasa est fortement déterminée par la politique des pesantren avec son kyai comme figure centrale.

L'effort fourni par les pesantren pour adopter un modèle moderne de l'éducation devait à l'origine faire face au problème de l'émergence de dualisme. Dans l'éducation. L'idéal est de pouvoir apporté une double éducation sous un même toit. Afin qu'il n'y ait pas de dichotomie dans le système d'éducation, ou ce qui est nommé l'éducation générale et l'éducation religieuse. Dans les développements ultérieurs, ces deux modèles d'enseignement sont gérés par deux organismes différents de la bureaucratie gouvernementale, le ministère de l'Éducation qui gère les établissements 
d'enseignement hérité des néerlandais, tandis que les madrasa et les pesantrens sont supervisés par le Ministère de la Religion. La différence entre les deux est ponctué de nouveau par la naissance de politiques gouvernementales de l'Ordre ancien favorise l'éducation publique plutôt que l'éducation religieuse. Cette situation s'est poursuivie jusqu'au commencement du gouvernement de l'ordre nouveau.

La politique gouvernementale en la matière est encore limitée aux efforts visant à renforcer la structure des madrasa. À cet égard, deux choses importantes ont été faites pour montrer l'attention portée par le gouvernement aux madrasa. Tout d'abord, formaliser les madrasa en essayant de convertir le statut de quelques madrasa de privé à public. Deuxièmement, restructurer les madrasa pour qu'ils soient en conformité avec les exigences de l'éducation nationale, en particulier avec l'uniformité et l'amélioration des programmes d'études utilisés (Fadjar, 1998).

Les niveaux de l'enseignement islamique en Indonésie plus précisément celui utilisé dans les madrasa, débute par:

1. Raudhatul $\mathrm{R} A$ (équivalent à l'école maternelle); c'est un établissement d'enseignement islamique qui éduque les enfants d'âge pré-scolaire, soit environ 4-6 ans. Le programme qui est enseigné dans Raudhatul RA porte plus sur les aspects du développement de la langue, émotionnel, cognitif et social. L'aspect de la foi commence à être introduit à travers l'enseignement de la religion .

2. Madrasa Ibtidaiyah (niveau élémentaire); c'est un établissement d'enseignement islamique qui éduque les enfants âgés de 6-12 ans. Le programme d'étude qui est enseigné en plus du programme général du Ministère de l'éducation nationale est un programme provenant du ministère des religions qui met l'accent sur l'apprentissage de la religion dont l'enseignement est divisé en Fiqh(les lois religieuses), Al Qur'an Hadits (le Coran et la parole du Prophète), l'histoire culturelle islamique et Aqidah Akhlak (la foi et la morale). D'une part, la séparation de l'apprentissage des matières religieuses permet un apprentissage plus spécifique pour mieux maitriser la matière, mais d'autre part cette séparation est un fardeau assez lourd pour les élèves du MI.

3. Madrasa Tsanawiyah (premier cycle du secondaire,collège); à savoir les établissements d'enseignement islamiques qui éduquent les enfants âgés de 13-15 ans. Comme pour le niveau du madrasa de l'école primaire, le programme d'enseignement qui est donné au MTs est une combinaison entre le programmes d'études du Ministère de l'éducation nationale et le programme du Ministère des religions. Bien sur, le volume de l'apprentissage des élèves du MTs est plus lourd que celui des élèves qui étudient au collège. Cependant, la combinaison du programme pour 
certains parents musulmans devient une alternative pour confier leurs enfants à un MTs car ils sont très occupés et ont donc peu de temps pour donner une éducation religieuse à leurs enfants à la maison.

4. Madrasa Aliyah (niveau secondaire, lycée) ; à savoir une institution éducative islamique qui éduquent les enfants âgés de 16-18 ans. Dans la deuxième année (c'est-à-dire l'équivalent de la première), comme les lycéens, les élèves doivent choisir une filière, soit sciences naturelles, sciences sociales, sciences religieuses islamiques ou les langues. A la fin de la troisième année (c'est-à-dire la terminale), les étudiants doivent passer un examen national qui déterminera leur réussite. Les diplômés de MA peuvent poursuivre leurs études dans des écoles publiques, des universités Islam, ou travailler directement. MA comme le lycée est appelé MA général ou professionnel, (pour le lycée technique, on l'appelle SMK) par exemple le MA professionnel (MAK) Le programme d'enseignement du MA est le même que le programme d'études du lycée, seulement au MA le programme de l'éducation islamique est plus complet. En plus d'enseigner des matières de l'école élémentaire, les étudiants ont des cours tels que: Fiqh(les lois religieuses), Al Qur'an Hadits (le Coran et les paroles du Prophète), l'histoire culturelle islamique et Aqidah Akblak (la foi et la morale) et l'arabe .

5. L'enseignement supérieur Islam (PTI); à savoir l'établissement d'enseignement islamique qui vise à devenir un centre d'étude et de développement de la science islamique qui est dirigé vers la création d'objectifs de formation, vise à préparer les étudiants à être des membres de la communauté qui ont des capacités académiques et professionnelles, qui sont capables de développer, diffuser et appliquer les connaissances de l'Islam, ainsi qu'améliorer le niveau de vie des gens et leur bien-être en les éduquant. Les établissements d'enseignement supérieur Islam comme IAIN/IAIS ou UIN offrent un large choix de filières, et sont devenues une alternative d'établissements supérieurs pour une partie de la communauté musulmane.

Le portrait des éducateurs dans les établissements d'enseignement islamiques, et en ce qui concerne notre étude les enseignants de madrasa ne sont pas encore tous professionnels, car beaucoup d'enseignants ne sont pas compétents. Le niveau de vie des enseignants qui est encore faible a aussi un impact sur la difficulté de trouver des enseignants qualifiés. Les modifications du programme d'enseignement 2013 portent, en substance, sur les changements dans le processus d'apprentissage et d'évaluation, changements qui exigent que les enseignants soient vraiment bien préparés. Plus précisément, ces changements peuvent être considérés comme un changement culturel et un changement de l'ambiance de l'enseignement qui 
commence du processus d'apprentissage jusqu'au système d'évaluation. Fondamentalement, le programme d'enseignement 2013 veut juste changer l'orientation de l'apprentissage des élèves qui au lieu de toujours mesurer les aptitudes scolaires (cognitives) des étudiants s'oriente vers le développement des attitudes et des compétences de base. Bien sûr, cela nécessite le développement d'une stratégie pour améliorer la qualité des enseignants des madrasa d'une manière planifiée, mesurée et ciblée, sinon, cela aura un impact sur la réalisation de la qualité, la pertinence et l'image des madrasa dans l'avenir.

Le système d'évaluation permet de mesurer les compétences des étudiants/ de l'enseignement à la sortie du madrasa qui à l'heure actuelle se réfère encore au système qui a été mis en œuvre par le gouvernement pour les madrasa et qui s'appelle US/M, tandis que pour les MT et MA, les étudiants doivent passer l'examen national UN. Ce système d'évaluation a beaucoup de défauts, car l'évaluation du processus d'apprentissage qui s'est déroulé pendant de nombreuses années depuis l'enseignement primaire et secondaire n'a pas de sens, car l'obtention du diplôme est uniquement déterminée par les 4 jours de l'examen national.

La fonction de l'évaluation, conformément à la loi 20 de 2003 , article 57, paragraphe 1 et 2, c'est "l'évaluation est effectuée afin de contrôler la qualité de l'éducation à l'échelle nationale comme une prise de responsabilité des acteurs de l'éducation envers les parties concernées." Mais en réalité, quand il est clair que certaines institutions ne répondent pas à la qualité de l'éducation exigée, comme en témoigne le nombre des étudiants qui n'ont pas réussi à l'examen, il n'y a pas d'ajustement ou de prise en charge pour améliorer le système d'enseignement de ces institutions.

La proportion des élèves qui suivent le primaire dans des madrasa varie selon les régions. Selon une analyse, on estime que dans $70 \%$ des villes/districts, environ $4 \%$ à $20 \%$ des élèves sont scolarisés dans des madrasa de l'école primaire au cycle du secondaire, dans quatre districts/ villes les écoles publiques accueillent environ la moitié du nombre des élèves, dans huit districts/villes, il n'y a aucun madrasa. Dans certaines communautés, le madrasa est une option, mais dans les zones reculées où le gouvernement n'a pas ouvert d'écoles publiques, les madrasa privés sont le seul type d'enseignement disponible.

Alors que le nombre d'étudiants dans les écoles publiques n'a pas augmenté au cours des dernières années, le développement des élèves dans les Madrasa a montré un taux de croissance encourageant. La croissance a atteint $2,5 \%$ pour les MI, $3,2 \%$ pour les MTs, et $9,4 \%$ pour les MA par an, pour répondre aux exigences de la demande en éducation de la population. La croissance est inégale entre les régions, il y a des provinces où la 
croissance est stagnante, mais il y a aussi une province dont la croissance a atteind 15\% par an (Hasanah, 2010, p. 8).

Les madrasa en tant qu'établissements d'enseignement islamique apportent une contribution significative à l'éducation publique en Indonésie, c'est pourquoi ils doivent être pris en compte dans les efforts de planification pour améliorer l'éducation en Indonésie avec les écoles publiques. Ainsi que les écoles publiques, la nécessité d'améliorer la qualité de l'éducation dans les madrasa doit passer par l'amélioration du processus d'enseignement, le perfectionnement du professionnalisme des enseignants, l'amélioration des infrastructures et leur utilisation, ainsi que l'utilisation des manuels et autre matériel pédagogique. L'étude a également révélée que l'éducation islamique fournit une base solide dans la formation du caractère de la nation à travers un processus d'apprentissage qui a été réalisé en accentuant le programme de l'enseignement religieux, le bon exemple de l'enseignant, ainsi que les habitudes qui ont été ancrées pendant la présence de l'élève à l'école.

\section{Portrait de l'enseignement islamique en France}

La République Francaise est un pays dont le territoire est situé en Europe de l'ouest, qui possède une frontière avec l'Angleterre (le tunnel sous la manche), ainsi qu'avec l'Espagne, l'Italie, la Suisse, l'Allemagne, le Luxembourg et la Belgique. Elle est baignée par la Manche, l'océan Atlantique et la mer Méditerranée. Néanmoins, la France possède aussi beaucoup de territoires à travers les océans du monde entier et a colonisé de nombreux pays et régions, y compris les pays avec une population à majorité musulmane, comme l'Algérie, le Maroc, la Tunisie et des pays d'Afrique. Dans ces États, la langue française est devenu la langue nationale, qui est utilisée dans les organismes gouvernementaux et à l'école. La population française se compose de diverses origines ethniques et religieuses. Les principales religions en France se composent des Catholiques, entre 83 et $88 \%$, les protestants $2 \%$, les juifs $1 \%$ et de 5 à $10 \%$ de musulmans (http://www.ladocumentationfrancaise.fr/dossiers/religionsfrance/panoram a religieux.shtml, téléchargé le 8 mei 2014).

Après la seconde guerre mondiale, le manque de ressources humaines pour reconstruire l'Etat français, pousse un grand nombre de musulmans à venir s'installer en France, en provenance de l'Afrique du Nord, et en particulier de l'Algérie. Les immigrants ont apporté leur culture et leur religion. C'est le début de changements importants et de l'apparition d'un nouveau dynamisme dans la pluralité des religions en France. Ce phénomène est confirmé par le Décret n ${ }^{\circ}$ 76-383 du 29 Avril 1976, sous la présidence de Valéry Giscard Estaing, sur «la loi du regroupement familial » qui permet aux ressortissants étrangers qui se sont installés en France pour le travail et qui 
vivent en France depuis plusieurs années, de faire venir leur famille. Comme de nombreux travailleurs étrangers sont musulmans, grâce à cette loi, leur nombre augmente de manière plus rapide car ils peuvent vivre en famille et avoir des enfants sur le sol français.

En 1985, a eu lieu une conférence islamique, financée par le Räbithah Alam Islämi (organisation islamique mondiale), où 141 pays musulmans ont participé et qui a abouti à la décision de créer la Fédération des musulmans de France. Cette Fédération a réussi à unifier 540 organisations réparties sur l'ensemble du territoire français et à protéger 1600 mosquées, ainsi que les établissements d'enseignement islamiques et les bâtiments islamiques en France. Les musulmans doivent pratiquer leur religion dans le cadre de la loi et le droit applicable en France. Ils sont autorisés à prier, jeûner, et à pratiquer les autres aspects de l'islam à condition que ce ne soit pas dans les lieux publics (http://www.vie-publique.fr/politiques-publiques/etat-culteslaicite/liberte-religieuse téléchargé le 8 mai 2014). Bien que les français ne souhaitent pas que la religion soit impliquée dans le gouvernement, (selon le principe séculaire) en 2002, le ministre de l'intérieur de l'époque, Nicolas Sarkozy a donné son accord pour la création d'un Conseil Francais du Culte Musulman/CFCM, qui est une organisation à but non lucratif et sans statut juridique spécial (Muslim in Europe, The economist, telecharge le 8 mai 2014).

Le développement de l'Islam en France est également décrit par le journaliste français qui est aussi un expert de l' Islam, Xavier Ternisien. Il a écrit que le développement de l'Islam en France est très rapide, cela se voit par l'apparition de nombreuses mosquées aux coupoles typiques, ce qui montre que les musulmans ne se cachent plus. Ce ne sont pas uniquement les mosquées qui poussent dans la capitale de la mode, mais aussi plusieurs écoles privées islamiques, dont le système d'éducation suit le système d'enseignement général français (http://fr.wikipedia.org/wiki/Conseil_ franc\%C3\%A7ais_du_culte_musulman, telecharge le 8 mai 2014).

En général, l'éducation en France est centralisée, c'est-à-dire qu'elle dépand du gouvernement. C'est pourquoi le Ministère de l'éducation nationale a un rôle très important à jouer sur l'ensemble du système éducatif . Le gouvernement français a également publié une loi rendant l'enseignement obligatoire jusqu'à l'âge de16 ans, ainsi que la mise en œuvre d'écoles publiques gratuites, de l'école primaire jusqu'au lycée.

Le système d'éducation en France est similaire au système de l'éducation en Indonésie. La principale différence réside dans le système d'évaluation, où en France le processus qui détermine si oui ou non un enfant peut entrer au collège ou au lycée n'est pas déterminé par un examen national, mais par les bulletins de notes et l'attitude de l'enfant pendant sa scolarité. 
Les étapes de l'éducation en France commence à partir de la maternelle jusqu' à l'université; 1) L'éducation préscolaire. L'école maternelle est un système d'éducation mis en œuvre comme une étape avant l'entrée à l'école primaire. L'éducation pré-scolaire commence à l'âge de 2 ans. L'attention de l'école pré-scolaire porte sur le développement physique, intellectuel et moral des enfants. Pour atteindre ces objectifs, le programme se compose d'exercices physiques, de jeux, de chants, de dessins et de peintures et de bricolages ou travail manuel; il y a aussi des exercices d'observation des objets présents dans l'environnement de l'enfant (http;//www.france.fr/etudier-enfrance/le-systerne-scolaire-francais-de-la-maternelle-au-lycee.html); L'enseignement primaire. L'école primaire en France débute à l'âge de 6 ans, et se déroule sur 5 ans, avec un maitre ou une maitresse par classe. L'école primaire est divisée en trois étapes, qui sont; (a) préparatoire, pour les enfants âgés de 6 à 7 ans; (b) élémentaire, pour les enfants âgés de 7 à 9 ans; (c) moyen, pour les enfants âgés de 9 à 11 ans.

Le programme de l'éducation primaire se compose de l'apprentissage de la langue française, la lecture et l'écriture, le calcul, l'histoire et la géographie, les sciences naturelles, le dessin, le travail manuel, le chant et le sport. (Tadjab, 1994, p. 100) Il n'y a pas d'examen national à la fin de l'école primaire en France. Les évaluations quotidiennes ne sont pas des choix multiples, mais toujours des essais, pour aider à former l'esprit critique des élèves. La notation des contrôles utilisent des lettres deA à D.

L'enseignement secondaire (Enseignement secondaire). L'enseignement secondaire en France est la continuation de l'enseignement primaire. L'enseignement secondaire est divisé en deux , à savoir le collège et le lycée (http;//www.france.fr/etudier-en-france/le-systerne-scolaire-francais -de-la-maternelle-au-lycee.html).

Le collège dure quatre ans. Le système de sélection des collégiens ne repose pas sur le NEM (note attribuée par un examen national), mais dépend du lieu d'habitation (zone) Les élèves étudient avec un professeur par matière, et changent de classe entre chaque cours. L'examen à la fin du collège (le brevet des collèges) porte sur trois matières, le français, les mathématiques et l'histoire/géographie. L'examen est national, mais c'est le rapport et les notes des élèves pendant les quatre années du collège qui décide si ils peuvent passer au lycée. Le brevet des collèges est considéré comme un entrainement pour le baccalaurérat.

L'enseignement secondaire supérieur (lycée) se réalise sur 3 ans. La sélection pour être admis à ce niveau est encore basée sur la zone (résidence), sauf pour les étudiants qui entrent à l'école professionnelle (SMK). Le lycée est gratuit et les étudiants peuvent emprunter des livres gratuitement. La formation professionnelle dans une forme limitée qui existe depuis 
longtemps. La division de l'enseignement professionnel fournit des experts dans le domaine de l'industrie et du commerce. En outre, il développe la formation professionnelle en offrant des programmes à temps partiel pour que les étudiants aient la possibilité de travailler tout en continuant à apprendre. L'examen final national du lycée est "le baccalauréat" qui détermine l'obtention du diplôme et qui fournit l'occasion aux élèves de poursuivre leurs études dans une université ou une grande école.

L'enseignement supérieur en France est divisé en deux, entre les Grandes Ecoles et les universités. En général, les grandes écoles ont un système de sélection plus rigoureux de sorte qu'elles sont considérées comme plus prestigieuses que l'Université. Une autre différence est que les Universités dépendent du Ministère de la Jeunesse, de l'Education nationale et de la recherche alors que les Grandes Ecole dépendent du Ministère de l'Industrie, en fonction du domaine enseigné. L'enseignement à l'Université est théorique et général. Alors que dans les Grandes Ecoles, l'enseignement est technique. L'éducation est très importante en France, et on peut lire dans le livre intitulé France, qu'en France il y a plus de 20 millions d'élèves, ce qui signifie près d'un quart de la population française. Les fonds destinés à l'éducation par le gouvernement français en 2004 se montent à environ 70 milliards de dollars (US), ce qui représente $23 \%$ du budget annuel total du gouvernement français (www.enseignementsup-recherge.gouv.fr).

Les professeurs sont bien payés en France, ce qui exige du professionnalisme, de sorte que la sélection pour être un enseignant est très serrée et ils doivent avoir un certificat professionnel. Cette hypothèse est étayée par une recherche qui explique que la profession d'enseignant est la troisième catégorie la mieux payée par le gouvernement. Au premier rang, on trouve les acteurs de la loi (juges/procureurs/avocats), puis les fonctionnaires (médecins et agents publiques), et au troisième rang, les personnels de l'enseignement (enseignants et inspecteurs de l'enseignement) (www.education.gouv.fr/cid1052/professeur-des ecoles.html,téléchargé le 8 mai 2014).

Les étapes de l'enseignement dans les écoles musulmanes sont les mêmes que les écoles publiques. En 2014, environ 2000 élèves ont fait leur rentrée dans 20 écoles musulmanes, tandis que 10 écoles sont encore en construction ou en attente de l'octroi de licences. Le nombre d'écoles musulmanes est encore faible par rapport aux écoles catholiques au nombre de 9000 et de 100 pour les écoles juives.

La majorité des écoles privées musulmanes sont dans une situation financière très critique, c'est pourquoi les écoles privées islamiques espèrent une coopération avec le gouvernement français pour que les salaires des enseignants soient pris en charge par le gouvernement. Cette coopération 
peut exister si les écoles religieuses suivent le programme d'enseignement national et ont de bons résultats. A ce jour, une seule école musulmane a réussi à obtenir des fonds du gouvernement, car cette école a réussi à obtenir $100 \%$ de réussite au bac, alors que le taux de réussite des écoles locales est de $81 \%$ de leurs élèves.

Le déclenchement de l'augmentation des écoles musulmanes ces 10 dernières années repose sur deux facteurs principaux. Le premier est le désir des parents musulmans qui viennent de la classe moyenne supérieure de donner une éducation de qualité à leurs enfants et aussi de leur présenter leur culture et leur religion à l'école. Le second est la loi n ${ }^{\circ}$ 2004-228 Du 15 mars 2004, qui interdit aux élèves des écoles publiques d'utiliser des attributs religieux, comme l'utilisation du voile. Les adolescentes ne sont pas autorisés à porter le voile quand elles pénètrent à l'intérieur de l'enceinte de l'école. Mais en dehors, elles sont libres de le porter. Cette loi à fait prendre conscience aux musulmans de l'importance d'une école musulmane pour les filles qui ne veulent pas être obligée de se découvrir pour pouvoir avoir accès à l'éducation. La France est un pays séculaire et laïc, où l'enseignement de la religion ne doit pas interférer dans les programmes des écoles publiques et les écoles publiques ne sont pas disposées à autorisé la pratique du culte, comme la prière, dans l'école.

L'éducation islamique en France est un ensemble d'enseignement/ d'écoles privées qui sont encore peu nombreuses. La première école privée islamique a été établie dans la zone de Vitrerie, dans une banlieue au sud de la France. Le programme est adapté au programme scolaire national, avec l'ajout d'un contenu local, à savoir l'enseignement de la langue arabe, l' instruction et la pratique religieuse de l'Islam. Education et Sauveur, est la deuxième école qui a ouvert ses portes à Aubervilliers, au nord de Paris. Une autre école privée musulmane, Ibn Rushd se trouve dans la ville française de Lille, au nord de la France et Al Kindi se situe dans la ville de Lyon. En Juillet 2003, le gouvernement français a approuvé la construction de La Réussite comme la première école secondaire musulmane de France (http;//www. leparisien.fr/societe/une,trentaine-d-etablishmentsmusulman-en-france-06-05-2013-2783929. php, téléchargé 7 mei 2014).

La propagation de l'islam en France augmente de manière constante. Pour renforcer l'islam dans ce pays, la Turquie aurait annoncé des plans pour construire un campus islamique dans la ville de Strasbourg. Son objectif est d'éduquer les musulmans français et de former de futurs imam.

"Nous allons constuire le campus le plus Islamique en Europe continentale», a déclaré Saban Kiper, au Conseil économique et social de la municipalité de Strasbourg, propos rapporté par le journal Libération du 
mercredi (9/10). Nos étudiants seront formés par les meilleurs imam et qualifiés pour transmettre les enseignements de l'Islam, a déclaré Kiper.

La construction d'un campus islamique en France est prévu pour être financée par la communauté musulmane française d'origine turque, qui a contribué au financement d'un montant de 15 millions d'euros. Le campus prévoit de former des imam pour combler le vide de chefs religieux musulmans en France. Actuellement, il n'y a que deux institutions privées qui forment les imam, les enseignants religieux ou universitaires, à savoir la Grande Mosquée de Paris et l'Union des organisations islamiques de France (UOIF).

La France a réussi à rendre l'école obligatoire et à fournir une éducation gratuite à tous les élèves, citoyens français et étrangers, à l'initiative de Jules Ferry depuis 1881. Jules Ferry a également construit des " Ecoles normales" pour éduquer des enseignants de qualité. Ces points sont une fierté pour les citoyens français, car depuis longtemps chaque enfant peut recevoir une éducation de qualité sans frais dans l'espoir que tous les enfants aient une chance égale pour devenir des citoyens performants (Error! Hyperlink reference not valid. 8 mei 2014).

\section{Comparaison de l'éducation islamique en Indonésie et un France.}

Une étude comparative est l'un des fondements qui renforcent la construction de la science (Arif, 2010). Les études comparatives menées en matière d'éducation peuvent être très significatives pour le développement de l'éducation dans une meilleure direction. La comparaison de l'éducation islamique en Indonésie et en France peut être considérée sous différents aspects;

1. Niveau de l'éducation. L'éducation en Indonésie et en France exigent l'entrée à l'école maternelle avant d'entrer dans l'éducation primaire. La durée de l'école primaire en France est de 5 ans, tandis qu'en Indonésie, à aussi bien au SD/MI elle dure 6 ans. A la fin de l'école primaire, les élèves doivent continuer à l'école secondaire. L'apprentissage dans l'enseignement secondaire en France se divise en deux, à savoir 4 années au collège et 3 ans au lycée, tandis qu'en Indonésie l' apprentissage est de 3 ans au SMP/MTs et de 3 ans au SMA/MA;

2. Le système éducatif. Le système éducatif en France, utilise un système centralisé qui est entièrement géré le gouvernement. Le gouvernement français a mis en œuvre une politique de l'enseignement obligatoire jusqu'à 16 ans avec la mise en œuvre d'un système scolaire gratuit pour chaque niveau d'enseignement. Alors que l'Indonésie, en raison de l'autonomie régionale qui utilise un système de décentralisation, le gouvernement laisse chaque région gérer les questions liées à l'éducation 
et aux établissements scolaires. La politique de l'enseignement obligatoire en Indonésie est de 9 ans si bien qu'à l'heure actuelle,l'école est gratuite pour les écoles primaires SD/MI et les collèges SMP/MTs. Il existe une différence de fond entre le système d'éducation islamique en France et en Indonésie. Les écoles islamiques en France sont des écoles privées dépendantes du Ministère de l'éducation nationale et ne reçoivent aucune aide du gouvernement, tandis qu'en Indonésie les madrasa sont des écoles religieuses privées ou publiques dépendantes du Ministère de la religion, et recoivent une variété d'aides financières ou matérielles;

3. Programme d'enseignement/Curriculum. Le programme d'enseignement en France est axé sur la maittrise des compétences de base approprié au développement des apprenants, l'enseignement religieux n'est pas autorisé dans les écoles publiques. Le programme d'enseignement des écoles musulmanes est adapté au programme général avec en plus des cours de langue arabe, de religion et des pratiques religieuses. Alors qu'en Indonésie, l'instruction religieuse est obligatoire dans toutes les écoles, qu'elles soient publiques ou religieuses, et spécialement dans les madrasa, le nombre d'heures d'instruction religieuse est plus important que dans les écoles publiques, avec l'ajout des cours de langue arabe, du Coran et de la parole du prophète, de l'histoire et de la culture islamique ainsi que l'enseignement de la morale;

4. Evaluation/Notation. L'évaluation dans les établissements d'enseignement français est effectuée de manière progressive, car il n'y a pas d'examen national à la fin de l'école primaire. Dans le secondaire de premier cycle, il y a un examen national (le Brevet des Collèges), mais l'admission au lycée restera dépendante des bulletins scolaires et du comportement tout au long de leur scolarité au collège. Le brevet des collèges est un examen pour s'entrainer au BAC, l'examen national qui a lieu à la fin du lycée et dont le résultat détermine la réussite des lycéens et dont l'obtention leur donne la possibilité de poursuivre leurs études à l'université.

L'évaluation en Indonésie est sanctionnée par examens nationaux depuis l'école primaire jusqu'à l'école secondaire, même si cette année, l'examen national est modifié en examen qui est fait au niveau provincial et l'obtention du diplôme est déterminée par l'école. Alors que l'examen national des SMP/MTs et SMA/MA reste centralisé.

Les contrôles en France n'ont pas la forme de questionnaire à choix multiples, mais la forme d'essais, pour aider les élèves à avoir l'esprit critique dans la résolution des problèmes. Alors qu'en Indonésie encore près de $80 \%$ des évaluations quotidiennes sont effectuées à partir de formulaires d'évaluation à choix multiples, l'essai représente un petit 
pourcentage et est plus censé mesurer la mémorisation que les capacités cognitives.

5. Le personnel de l'enseignement. Les éducateurs / enseignants en France sont des professionnels qui ont été recrutés par le biais d'un processus de sélection rigoureux. La profession d'enseignant en France est très prisée. C'est tout à fait compréhensible, car le bien-être des enseignants est assez élevé. L'engagement du gouvernement français attribue environ $23 \%$ du budget annuel du gouvernement à l'éducation et permet de fournir un revenu raisonnable pour les enseignants. Alors qu'en Indonésie, la profession d'éducateurs / enseignants est moins recherchée en raison du niveau des professeurs, qui est encore moyen, entre 2 et 5 millions de roupies pour les enseignants qui sont fonctionnaires, mais pour les enseignants de madrasa dans les régions, le salaire est encore parfois inférieur à un million de roupies. Le poste d'enseignant n'est donc pas très désiré et entraine des difficultés pour effectuer une sélection rigoureuse pour les postes de professeur.

\section{CONCLUSIONS}

Avec le développement de l'Islam en France, le nombre de lieux de culte et les activités islamiques sont en augmentation. Ce ne sont pas seulement les mosquées qui se développent rapidement actuellement, mais également les établissements d'enseignement islamiques dans le pays sont en forte croissance. Un certain nombre d' écoles islamiques ont ouvert en France . La première école privée islamique a été établie dans la zone de Vitrerie, dans une banlieue au sud de la France. Le programme est adapté au programme scolaire national, avec l'ajout d'un contenu local, à savoir l'enseignement de la langue arabe, l' instruction et la pratique religieuse de l'Islam. La construction d'un campus islamique en France est prévu pour être financé par la communauté musulmane française d'origine turque, qui contribuerait au financement d'un montant 15 millions d'euros. Le campus prévoit de former des imam pour combler le vide de chefs religieux musulmans en France. Actuellement, il n'y a que deux institutions privées qui forment les imam, les enseignants religieux ou universitaires, à savoir la Grande Mosquée de Paris et l'Union des organisations islamiques de France (UOIF). La France a réussi à rendre l'école obligatoire et à fournir une éducation gratuite à tous les élèves, citoyens français et étrangers, à l'initiative de Jules Ferry depuis 1881. Jules Ferry a également construit des " Ecoles normales" pour éduquer des enseignants de qualité. Ces points sont une fierté pour les citoyens français, car depuis longtemps chaque enfant peut recevoir une éducation de qualité sans frais dans l'espoir que tous les enfants aient une chance égale pour devenir des citoyens performants. En regardant 
l'évolution rapide des institutions islamiques en France, même si leur nombre est encore faible, l'enseignement islamique est organisé avec un standard élevé en conformité avec les normes de l'école en général en France. En ce qui concerne l'Indonésie, les établissements d'enseignement islamiques ont participé de manière historique au développement de la nation indonésienne. L'éducation islamique en Indonésie a contribué de façon très significative à l'enseignement au niveau national, aussi bien sur l'aspect conceptuel que pratique de l'éducation. L' éducation, en général est influencée par le concept et l'esprit des valeurs islamiques. Mais d'autre part, le système d'éducation islamique en Indonésie à ce jour nécessite encore d'une innovation constante, ainsi que du soutien du gouvernement. En terme de quantité, le nombre d'écoles islamiques en Indonésie est très important, mais leur organisation est encore variée et la majorité ne remplit pas les standards de la norme nationale sur l'éducation(SNP). Par conséquent, la quantité devrait être directement proportionnelle à la qualité de l'enseignement, de sorte que les objectifs pédagogiques puissent être atteints de manière efficace. C'est la remarque apportée par cette étude comparative.

\section{BIBLIOGRAPHY}

An-Nahlawi, Abdurrahman. (1878). Ushül at-Tarbiyah al-Islamiyah wa Asälïbuba fi al-bayt wa al-madrasah wa al-Mujtama', Damaskus: Dar al-Fikr.

An-Nahlawi, Abdurrahman. (1989). Prinsip-prinsip dan Metoda Pendidikan Islam, terj. Herry Noer Ali, Bandung: CV. Diponegoro.

Arifin, Muzayyin. (1993). Filsafat Pendidikan Islam, Jakarta: Bumi Aksara.

Asy-Syaibany, Omar Mohammad al-Toumy. (1979). Falsafab Pendidikan Islam, terj. Hasan Langgulung, Jakarta: Bulan Bintang.

Azra, Azyumardi. (1999). Pendidikan Islam, Tradisi dan Modernisasi Menuju Millenium Baru, Jakarta: Logos Wacana Ilmu.

Djojonegoro, Wardiman. (1996). 50 Tahun Perkembangan Pendidikan

Dhofier, Z.(1982). Tradisi Pesantren.Jakarta: LP3ES.

Fadjar, M.A. (1998). Madrasah dan Tantangan Modernitas. Bandung: Mizan.

Hasanah, Aan. (2014). Pemberdayaan Madrasah (Perspektif Teori Sosial Kritis), makalah, Bandung.

Langgulung, Hasan. (1986). Manusia dan Pendidikan: Suatu Analisa Psikologi dan Pendidikan, Jakarta: Pustaka Al-Husna.

Muhadjir, Noeng. (1987). Ilmu Pendidikan dan Perubahan Sosial, Suatu Teori Pendidikan, Yogyakarta; Rake Sarasin.

Mohamad, Adriyant. Pembiayaan Pendidikan Vokasi dan Contoh Kasus Beberapa Negara, mei 2012

Nasution, S.(1994). Azas-azas Kurikulum, Bandung: Jemmars.

Nazir, Mohammad. (2005). Metode Penelitian. Ghalia Indonesia. Bogor. 
Nurkamri.blogspot.com/.../perbandingan-sistem-pendidikan, diakses 11 Mei 2014.

Qutb, Muhammad. (1984). Sistem Pendidikan Islam, Bandung: Remaja Rosda Karya.

Rahman, Fazlur. (1982.) Islam and Modernity, Chicago: The University of Chicago Press.

Rahman, Musthofa. (2001). "Pendidikan Islam dalam Perspektif Al-Qur'an", dalam Ismail SM (ed.), Paradigma Pendidikan Islam, Yogyakarta: Pustaka Pelajar.

Rohman, Arif. (2010). Pendidikan Komparatif. Yogyakarta: Laksbang Grafika Sudjana, Nana. (1995). Dasar-dasar Proses Belajar-Mengajar, Bandung: Sinar Baru Algesindo, 1995.

Suwito. (2004). Filsafat Pendidikan Akblak Ibnu Miskawaih, Yogyakarta: Belukar, Cet. I.

Tafsir, Ahmad. (1992). Ilmu Pendidikan dalam Perspektif Islam, Bandung: Remaja Rosda Karya.

Tafsir, Ahmad., (ed.), (n.d) Epistemologi Ilmu Pendidikan Islam, Bandung: Remaja Rosda Karya dan Fak. Tarbiyah IAIN Sunan Gunung Djati, .

Tafsir, Ahmad. (1994). Kajian Ilmu Pendidikan Islam di LAIN, pidato pengukuhan guru besar Madya Ilmu Pendidikan Islam, IAIN Bandung 24 November, 1999.

Tadjab. (1994). Pendidikan Islam, Surabaya: Karya Abditama

Susanto, dkk. (2010). Pendidikan Nasional Pendidikan Pada Periode 1962-1968. Dalam makalah Pendidikan Nasional 2010, diunduh pada 1 mei 2014

Yuandakusuma, www.slideshare.net/ yuandakusuma/Islam_di_ Perancis, diunduh pada 7 mei 2010

http://www.ladocumentationfrancaise.fr/dossiers/religions-

france/panorama-religieux.shtml, téléchargé le 8 mei 2014

http://www.vie-publique.fr/politiques-publiques/etat-cultes-laicite/libertereligieuse téléchargé le 8 mei 2014

http://fr.wikipedia.org/wiki/Conseil_franc $\%$ C3\%A7ais_du_culte_musulma $\mathrm{n}$, telecharge le 8 mei 2014

http;//www.france.fr/etudier-en-france/le-systerne-scolaire-francais-de-lamaternelle-au-lycee.html

http;//www.france.fr/etudier-en-france/le-systerne-scolaire-francais-de-lamaternelle-au-lycee.html

www.enseignementsup-recherge.gouv.fr

http://www.education.gouv.fr/cid1052/professeur-des-ecoles.html, téléchargé le 8 mei 2014

http;//www.leparisien.fr/societe/une_trentaine-d-etablishments-musulmanen-france-06-05-2013-2783929.php, téléchargé 7 mei 2014, 8 mei 2014 